(1)

CrossMark

\title{
The International Collaborative Effusion (ICE) database: an ERS Clinical Research Collaboration
}

\author{
Rahul Bhatnagar ${ }^{1}$, Julius Janssen $\mathbb{0}^{2}$ and Nick Maskell ${ }^{1}$ \\ Affiliations: ${ }^{1}$ Academic Respiratory Unit, University of Bristol, Bristol, UK. ${ }^{2}$ Pulmonology Dept, Canisius- \\ Wilhelmina Ziekenhuis, Nijmegen, The Netherlands. \\ Correspondence: Rahul Bhatnagar, Academic Respiratory Unit, Learning and Research Building (Level 2), \\ Southmead Hospital, Bristol, BS10 5NB, UK. E-mail: Rahul.Bhatnagarlabristol.ac.uk
}

@ERSpublications

The ICE database project is a new ERS Clinical Research Collaboration aiming to establish and improve multinational projects in pleural disease http://bit.ly/2G4FvgO

Cite this article as: Bhatnagar R, Janssen J, Maskell N. The International Collaborative Effusion (ICE) database: an ERS Clinical Research Collaboration. Eur Respir J 2019; 53: 1900591 [https://doi.org/10.1183/ 13993003.00591-2019].

\section{Background}

Pleural medicine is now well-established as an important subspecialty of respiratory medicine, with pleural effusions alone estimated to affect over 2 million people per year in Europe [1]. The range of pleural conditions is broad, encompassing those which are malignant or benign; treatable and requiring palliation; and acute or chronic. Interventions such as thoracoscopy and indwelling pleural catheters play a key role in the pleural specialist's arsenal, as does the increasingly ubiquitous thoracic ultrasound. Indeed, technological improvements have not only fuelled the ultrasound revolution but have also led to developments in other forms of diagnostic imaging and testing specifically geared towards the patient with a pleural condition [2].

To coincide with the above, over the past decade there has been a surge in the number of major studies, many of them randomised controlled trials, focusing on conditions such as malignant pleural effusion, mesothelioma and pleural infection [3-8]. The results of these have had an immediate and practicechanging effect on an international scale, even going so far as to change how entire treatment options are offered to patients in some centres [4], demonstrating an ongoing hunger for the highest quality evidence base amongst respiratory physicians with an interest in pleural disease. This wave of robustly designed, sufficiently powered studies and trials will hopefully continue over the coming years.

There are, however, significant challenges to delivering clinically orientated research in those with pleural disease. Conditions such as pleural infection and pneumothorax tend to be identified and require treatment in the acute setting, minimising the opportunity for study involvement. Additionally, those with malignant pleural disease are often highly comorbid and/or in need of palliative measures, once again restricting the ability to enrol sufficient numbers into trials; recent data have shown that, in some cases, despite large numbers of patients being screened, the number who actually progress to randomisation or treatment allocation can be surprisingly low [3]. Finally, in comparison to other conditions which sit under the wider respiratory umbrella (such as pulmonary hypertension), research into pleural disease is relatively underfunded, a fact in no small part down to a lack of true understanding of pleural pathophysiology and thus the possibility of novel pharmacological treatment targets. 
In many cases, the key to overcoming some of these hurdles is wider collaboration, broadening the pool of potential participants to compensate for any individual site's local challenges to obtaining data. This ethos is particularly vital when one accepts that, in addition to those detailed above, there are a range of important pleural conditions which are rarer or harder to classify, with the management and knowledge of many of these conditions founded upon single-centre case series only. They nonetheless remain clinically relevant to many general respiratory practitioners, for example due to a tendency to require recurrent intervention or because there exists a theoretical risk of progression to a more significant or malignant condition [9].

\section{A pleural clinical research collaboration}

Recognising the limitations of single-centre work, there is a strong desire to contribute to larger-scale projects amongst pleural-interested physicians $[10,11]$. In an attempt to leverage this, the Thoracic Oncology Assembly of the European Respiratory Society (ERS) have supported the creation of the International Collaborative Effusion (ICE) database as part of the Clinical Research Collaboration (CRC) scheme [12].

The aims of the ICE database are to establish consistent, effective working amongst pleural practitioners on a scale never-before attempted, leading to wider participation in important pleural research and, potentially, to lay the groundwork for more ambitious, multinational projects. The intended focus of the database is primarily on those conditions which are "under-represented" in the pleural literature, with a view to creating a foundation upon which practice-changing studies in these areas may be designed.

\section{The ICE database to date}

The collaboration was inaugurated in 2017, with co-ordination and hosting provided by the Academic Respiratory Unit in Bristol, UK, who have a strong background in delivering multicentre pleural studies of various designs. Initial membership was drawn from the ERS pleural interest group, who meet on at least a yearly basis at the ERS International Congress. At the time of writing there are 16 sites across 11 European countries, as well as South Africa, who have agreed to participate in the initial stages of the project, although the potential exists for this number to increase dramatically if the early stages of the project are felt to be successful. We would encourage those who may wish to contribute to the CRC to make contact with the corresponding author.

From the outset, the ICE collaboration has placed the democratic process and group collaboration at the centre of its work, taking the view that having greater input and "ownership" of a project for all sites, small and large, will lead to both better engagement with data entry and with any future undertakings. As a first step in this, discussions were had as to the general format of the data which would be collected. It was decided that focusing on a series of simultaneous, large scale retrospective studies would be the most effective starting point for the collaboration, doing so in a way that would create a platform for future prospective data collection projects. Data would be entered by each site directly onto a centralised research database, which would be designed in such a way that expansion to additional projects or revisiting established ones would be possible.

Following this, all members of the CRC were asked to provide a list of up to three topics that they felt to be important and worthy of further study. A total of 17 topics were suggested, spanning the breadth of pleural conditions and challenges, before being voted on by the whole group to determine what the ICE database would focus on. The four most voted-for disease areas were: chylothorax, eosinophilic effusions, non-specific pleuritis, and recurrent non-malignant effusions caused by organ failure (e.g. hepatic hydrothorax). Examination of the literature demonstrates that these topics, as well as being of clear clinical interest, are in need of more robust data to support practice. The largest series relating to non-malignant pleural effusions (not limited to those related to organ failure) contained 356 patients over 7 years [13]. For chylothorax, the equivalent is another study that described the outcomes of 203 patients over 21 years [14]. Eosinophilic effusions and non-specific pleuritis have been addressed in studies with only 135 and 208 patients, respectively $[15,16]$. Almost all series, including those listed here, are limited by drawing data from single centres.

The next stage was to identify and agree research questions, along with the more specific data points which sites would be asked to provide for each condition (see table 1). Priority was given to finding a balance between collecting robust datasets without overburdening those busy clinicians who would be tasked with finding and entering the information. By whole-group agreement, small (two- or three-person) "topic subgroups" were formed, with each of these leading on identifying relevant research questions as well as suggesting specific data points to be gathered (see table 1). Once each subgroup had completed this, there ensued two rounds of comment, review and adjustment by the wider team, before the final datasets were confirmed. At the time of writing, the trial database is in the later stages of being built and tested, using the product of the efforts of the whole pleural CRC as a template. 
TABLE 1 Initial International Collaborative Effusion (ICE) database subtopics and associated research questions

\section{Clinical research collaboration subtopic}

Chylothorax

Eosinophilic pleural effusion (EPE)

Non-specific pleuritis (NSP)

Non-malignant effusions (NME) secondary to cardiac, renal or hepatic dysfunction

\section{Selected important research questions}

What are the common presenting features of chylothorax?

What are the commonest causes of chylothorax?

How is chylothorax typically/best investigated?

What treatments are typically required/used for chylothorax?

What are the negative outcomes associated with chylothorax?

What is the incidence of EPE?

Is there an association between EPE and systemic eosinophilic diseases?

What is the prevalence of malignant pleural effusion in patients with EPE?

What are the most effective diagnostic procedures in EPE?

What proportion of EPEs are related to earlier pleural diseases/interventions (e.g. spontaneous pneumothorax, an earlier diagnostic thoracentesis)?

What is the prevalence and what agents are associated with drug-induced eosinophilic pleural effusion?

What are the commonest causes of NSP?

How many patients with an initial diagnosis of NSP develop malignancy during subsequent follow-up?

Is development of malignancy (false-negative NSP) related to thoracoscopic technique or difficulty?

What is the minimal safe follow-up time of NSP to exclude malignancy as eventual diagnosis?

What percentage was probably infective in nature, and what were the causes, if identified?

What are the baseline characteristics of patients who develop NME?

What are the prognostic features of NME?

What are the features of refractory NME?

What treatments (medical and interventional) are typically required for NME?

What are the complications with pleural intervention in patients with NME?

\section{Moving forward}

Once finalised, the ICE database will be used to generate what we anticipate being the largest series ever published for each of the four topics in question. Data entry is expected to begin in the summer of 2019. Analysis will be led by each of the small topic groups, with each manuscript being made available for review by each CRC member, cementing the collegiate nature of this project. Our hope is that this early work can act as proof that the concept of sustained, large, multinational collaborations in pleural conditions can be both feasible and successful.

The longer-term ambition for the ICE database goes further, with it potentially being used to rapidly generate future cohorts for other low-incidence pleural conditions. Additionally, if the challenges of negotiating local ethics applications can be overcome, we will also be able to undertake even more robust, prospective projects, all using the same platform. In fact, we believe that the ICE database and pleural CRC may become the underpinnings of a large-scale (potentially industry-supported) biobank, with such an entity potentially able to offer the greater insights into the pathophysiology of pleural disease that are desperately needed to drive the next generations of pleural research questions and improvements in patient care.

Acknowledgements: The authors would like to thank the following for their contribution to the ICE database so far: Marios Froudrakis, Stavros Anevlavis (Alexandropoulos, Greece); Ales Rozman (Golnik, Slovenia); Arnaud Scherpereel (Lille, France); Charles Marquette (Nice, France); Philippe Astoul (Marseille, France); Jose Porcel (Lleida, Spain); Uffe Bodtger (Naestved, Denmark); Najib Rahman (Oxford, UK); John Corcoran (Plymouth, UK); Mohammed Munavvar (Preston, UK); Steven Walker, Alison Horne (Bristol, UK); Coenraad Koegelenberg, Jane Shaw (Cape Town, South Africa); Peter Driesen (Turnhout, Belgium); Magdalena Grabczak, Piotr Korczynski, Rafal Krenke (Warsaw, Poland).

Conflict of interest: None declared.

\section{References}

1 Sahn SA. Pleural effusions of extravascular origin. Clin Chest Med 2006; 27: 285-308.

2 Heffner JE, Klein JS, Hampson C. Diagnostic utility and clinical application of imaging for pleural space infections. Chest 2010; 137: 467-479.

3 Bhatnagar R, Keenan EK, Morley AJ, et al. Outpatient talc administration by indwelling pleural catheter for malignant effusion. N Engl J Med 2018; 378: 1313-1322. 
4 Davies HE, Mishra EK, Kahan BC, et al. Effect of an indwelling pleural catheter versus chest tube and talc pleurodesis for relieving dyspnea in patients with malignant pleural effusion: the TIME2 randomized controlled trial. JAMA 2012; 307: 2383-2389.

5 Zalcman G, Mazieres J, Margery J, et al. Bevacizumab for newly diagnosed pleural mesothelioma in the Mesothelioma Avastin Cisplatin Pemetrexed Study (MAPS): a randomised, controlled, open-label, phase 3 trial. Lancet 2016; 387: 1405-1414.

6 Clive AO, Jones HE, Bhatnagar R, et al. Interventions for the management of malignant pleural effusions: a network meta-analysis. Cochrane Database Syst Rev 2016; 5: CD010529.

7 Maskell NA, Davies CW, Nunn AJ, et al. UK controlled trial of intrapleural streptokinase for pleural infection. N Engl J Med 2005; 352: 865-874.

8 Rahman NM, Maskell NA, West A, et al. Intrapleural use of tissue plasminogen activator and DNase in pleural infection. N Engl J Med 2011; 365: 518-526.

9 Davies HE, Nicholson JE, Rahman NM, et al. Outcome of patients with nonspecific pleuritis/fibrosis on thoracoscopic pleural biopsies. Eur J Cardiothorac Surg 2010; 38: 472-477.

10 Muruganandan S, Azzopardi M, Fitzgerald DB, et al. Aggressive versus symptom-guided drainage of malignant pleural effusion via indwelling pleural catheters (AMPLE-2): an open-label randomised trial. Lancet Respir Med 2018; 6: 671-680.

11 Wahidi MM, Reddy C, Yarmus L, et al. Randomized trial of pleural fluid drainage frequency in patients with malignant pleural effusions. The ASAP Trial. Am J Respir Crit Care Med 2017; 195: 1050-1057.

12 Brightling C, Genton C, Bill W, et al. ERS Clinical Research Collaborations: underpinning research excellence. Eur Respir J 2018; 52: 1801534.

13 Walker SP, Morley AJ, Stadon L, et al. Nonmalignant pleural effusions: a prospective study of 356 consecutive unselected patients. Chest 2017; 151: 1099-1105.

14 Doerr CH, Allen MS, Nichols FC 3rd, et al. Etiology of chylothorax in 203 patients. Mayo Clin Proc 2005; 80: 867-870.

15 Krenke R, Nasilowski J, Korczynski P, et al. Incidence and aetiology of eosinophilic pleural effusion. Eur Respir J 2009; 34: 1111-1117.

16 Janssen JP, Ramlal S, Mravunac M. The long-term follow up of exudative pleural effusion after nondiagnostic thoracoscopy. J Bronchology 2004; 11: 169-174. 